\title{
Temporal and along-shelf distribution of the larval fish assemblage at Gran Canaria, Canary Islands
}

\author{
MARTA MOYANO and SANTIAGO HERNÁNDEZ-LEÓN \\ Laboratorio de Oceanografía Biológica. Facultad de Ciencias del Mar. Universidad de Las Palmas de Gran Canaria, \\ Campus Universitario de Tafira, 35017 Las Palmas de Gran Canaria, Canary Islands, Spain. \\ E-mail: marta.moyano101@doctorandos.ulpgc.es
}

\begin{abstract}
SUMMARY: Temporal and spatial variations of the larval fish community off the island of Gran Canaria (Canary Islands) were studied in weekly surveys from October 2005 to June 2006. A total of 156 taxa, belonging to 51 families and 15 orders, were identified. Myctophidae was by far the most abundant family (30\%), followed by Sparidae (11\%), Clupeidae (9\%) and Gonostomatidae (7\%). As expected for an oceanic island, neritic and oceanic taxa contributed in similar proportions. Leeward and windward retention areas were found for total egg and neritic larval abundance. However, seasonality showed a stronger influence on the annual larval assemblage than sampling site, as the latter was not significant on a long time scale. Results suggest that there are two seasonal larval assemblages corresponding to the two main characteristic periods of the water column in these waters: mixing (winter) and stratification (summer). In addition, a significant relationship was recorded between lunar illumination and small mesozooplankton biomass, suggesting that this relationship may be extended to certain neritic families. The most abundant neritic larvae (Sparidae) showed this lunar pattern, which partially supports a recent hypothesis about the effect of lunar illumination on larval fish survival and development in subtropical waters.
\end{abstract}

Keywords: ichthyoplankton, larval assemblages, mesozooplankton, seasonal variation, lunar illumination.

\begin{abstract}
RESUMEN: VARIACIONES ESTACIONALES Y ESPACIALES DE LA COMUNIDAd DE LARVAS DE PECES DE GRAN CANARIa, Islas CANARIAS. - Se estudiaron las variaciones temporales y espaciales en la comunidad de larvas de peces de la isla de Gran Canaria (Islas Canarias) a partir de muestreos semanales desde octubre 2005 a junio 2006. Se identificaron un total de 156 taxones, pertenecientes a 51 familias y 15 órdenes. La familia Myctophidae fue la más abundante (30\%), seguida por Sparidae (11\%), Clupeidae (9\%) y Gonostomatidae (7\%). Como es de esperar en una isla oceánica, los taxones neríticos y oceánicos contribuyeron en proporciones similares. Se encontraron dos zonas de retención para huevos y larvas de especies neríticas, a sotavento y barlovento de la isla. Sin embargo, la estacionalidad mostró una mayor influencia en la estructura de la comunidad larvaria que la zona de muestreo, siendo ésta no significativa a largo plazo. Se observaron dos asociaciones estacionales de larvas correspondiendo con los dos períodos más característicos en la columna de agua: periodo de mezcla (invierno) y de estratificación (verano). Además, se encontró una relación significativa entre la iluminación lunar y la biomasa del zooplancton de pequeño tamaño, que podría ser extensible a las larvas neríticas. Las larvas neríticas más abundantes (Sparidae) mostraron una relación con el ciclo lunar, apoyando parcialmente una hipótesis reciente sobre el efecto de la iluminación lunar en la supervivencia larvaria y su desarrollo en aguas subtropicales.
\end{abstract}

Palabras clave: ictioplancton, asociaciones de larvas, mesozooplancton, variaciones estacionales, iluminación lunar.

\section{INTRODUCTION}

In an attempt to understand population dynamics of marine fishes, studies have historically focused on the growth and survivorship of their early life stages (Hjort, 1914; Houde, 1987, 2008), as it is during these pre-recruitment stages that the success of a year class is determined. In the particular environment of oceanic islands, fish larvae from local populations need to avoid advection. Physical retention (Boehlert et al., 1992; Cowen and Castro, 1994) and larval behaviour (Paris and Cowen, 2004; Leis, 2007) are 
involved in the maintenance of larval fish populations close to shore, a phenomenon known as "conservation" (Leis, 1982). Moreover, self-recruitment, dependent upon larval retention, is considered to be the main factor sustaining local fish populations (Jones et al., 1999; Swearer et al., 2002). Nevertheless, in certain areas larval inputs from the outside may also be necessary to maintain these populations (Cowen et al., 2006).

Gran Canaria is one of the major islands of the Canary Archipelago, and is located in the NE Atlantic at latitude $28^{\circ} \mathrm{N}$. The proximity of this archipelago to the NW African coastal upwelling determines its unique environmental conditions. The interaction between the Canary Current flowing through the islands, the coastal upwelling and Trade Winds creates an area of high mesoscale activity (Barton et al., 1998). Thus, warm wakes and eddies are generated south of the Islands (Arístegui et al., 1994; Sangrá et al., 2007), and upwelling filaments frequently reach the shores of the easternmost islands of the archipelago (La Violette, 1974; Pacheco and HernándezGuerra, 1999). The influence of these mesoscale oceanographic structures on the ichthyoplankton off Gran Canaria Island was first described by Rodríguez et al. (2001). These authors identified two retention areas for eggs and neritic larvae: 1) upstream (north-northeast) where the weak inflowing Canary Current separates as it impinges on the island, and 2) downstream (south-southwest) where a warm lee is generated. This pattern has also been recently proposed for neritic decapod larvae (Landeira et al., 2009). High values of mesozooplankton biomass have also been recorded in this warm lee (Rodríguez et al., 2001; Hernández-León, 1988).

Despite the higher productivity found in the warm lee and the frequent influence of upwelling filaments, the Canary waters are typically oligotrophic (De León and Braun, 1973; Braun, 1980). During most of the year, the presence of a seasonal thermocline restrains the vertical flux of nutrients up to the surface, limiting phytoplankton growth (Arístegui et al., 2001). This thermocline is eroded during winter due to surface cooling, causing the mixed layer to reach its maximum depth (Barton et al., 1998). During this short mixing period, phytoplankton can grow faster and bloom: the late winter bloom of subtropical regions (De León and Braun, 1973; Braun, 1980). Mesozooplankton doubles its biomass during this late winter bloom (Hernández-León 1988; Hernández-León et al., 2004). However, the influ- ence of these seasonal variations on the larval fish assemblage off the Canary Islands is still unknown.

Few studies have dealt with the taxonomic composition of the whole larval fish community in the area (Rodríguez, 2000; Rodríguez et al., 1999, 2001). Furthermore, most of these studies consisted in short-time surveys and their sampling stations were either located in the oceanic region or near the coastal upwelling. Only Bécognée et al. (2006) carried out an annual study in shallow waters off Gran Canaria, but their study only focused on clupeoid and scombrid larvae. Therefore, the main goal of this work was to analyze the temporal and horizontal variations of the ichthyoplankton distribution and composition off Gran Canaria. In addition, the interactions between the ichthyoplankton and the biophysical environment were studied. The use of these data for determining species spawning periods and grounds was examined.

\section{MATERIAL AND METHODS}

Weekly sampling was carried out from October 2005 to June 2006 during daylight hours onboard the R.V Solana II at the eastern and southern flanks of Gran Canaria (Fig. 1). Five sampling stations, 10 nautical miles apart, were located over the $100 \mathrm{~m}$ isobath. CTD casts were performed to obtain vertical profiles of temperature, salinity and fluorescence using a SBE25 (Sea-Bird Electronics Inc., Bellevue, WA, USA). Phytoplankton chlorophyll $a$ (Chl $a$ ) was derived from vertical profiles of in-situ fluorescence, calibrated with samples collected at $15 \mathrm{~m}$ with a Niskin bottle. These samples of $500 \mathrm{ml}$ of seawater were filtered through Whatman GFF filters and preserved in liquid nitrogen. Then, chlorophyll $a$ concentrations were measured by the flourimetric method (Yentsch and Menzel, 1963).

Plankton samples were taken with oblique Bongo net tows down to $90 \mathrm{~m}$ depth and at a speed of about 2-3 knots. Nets were fitted with $200 \mu \mathrm{m}$ mesh and with a flowmeter (General Oceanics) to measure the volume of filtered water. The first sample was fractionated with a $1000 \mu \mathrm{m}$ mesh to quantify small (200 to $1000 \mu \mathrm{m})$ and large mesozooplankton (>1000 $\mu \mathrm{m})$ biomass as dry weight, following the method of Lovegrove (1966). The second sample was quickly preserved in $4 \%$ buffered formaline for further taxonomical analyses. Once in the laboratory, all fish larvae were sorted. Larvae were identified to the 


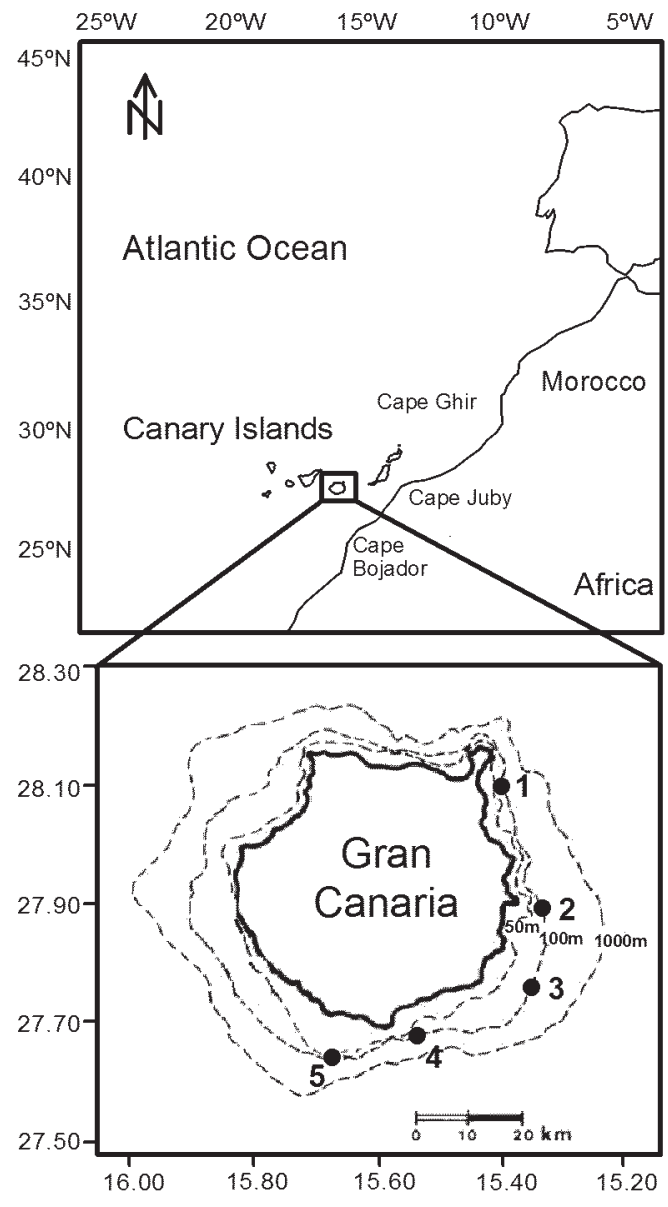

FIG. 1. - Map of the study area showing the location of the five sampling stations over the Gran Canaria shelf, Canary Islands (northeast subtropical Atlantic).

lowest taxonomic level possible using a dissecting microscope (Leica MZ 9.5). Fish eggs were sorted from November 2005 to June 2006 but only Clupeidae eggs were identified.

Greenwood classification (CLOFETA, Queró et $a l .$, 1990) was used for taxonomical organization of fish larvae. Following Rodriguez et al. (1999), these larvae were divided into three categories (Neritic, Oceanic and Other) in relation to adult behaviour.

In order to analyze the relationships between environmental variables and larval abundance, Spearman's rank correlation was carried out. Differences in ichthyoplankton abundance and mesozooplankton biomass among stations and months were analyzed using the non-parametric analysis of variance for repeated measures (Friedman test). To assess the variability of the mesozooplankton and larval fish abundance through the lunar cycle, standardization was performed considering the maximum value in abundance for each complete lunar cycle as $100 \%$.
Then, Kruskal-Wallis ANOVA (K-W ANOVA) was carried out on the standardized variables to analyze differences among the four lunar phases centred in the new, crescent, full and waning moon. Parametric tests were not used because most of the variables did not meet the underlying conditions of normality (Kolmogorov-Smirnov) and homogeneity of variances (Levene's test). All statistical procedures were performed using the Statistica 7.0 software package (StatSoft Inc., 2006).

In addition, a two-way analysis of similarities (ANOSIM, Primer software, Clarke and Warwick, 2005) was conducted to test for significant differences in the species composition among months (averaged accross sites), as well as diferences in the species composition among sites (averaged accross months). Only species contributing at least $5 \%$ to the total abundance were considered. Prior to generating the Bray Curtis resemblance matrix to perform the ANOSIM procedure, data were square-root transformed to reduce the weighting of dominant species. Temporal variability was analyzed on a monthly basis to reduce biasing due to the effect of plankton patchiness.

\section{RESULTS}

\section{Oceanographic conditions and ichthyoplankton and zooplankton distribution}

Environmental variables followed the typical annual cycle in the Canary Island waters (Fig. 2). During October and November, the water column was well stratified with surface temperatures around $23^{\circ} \mathrm{C}$ (Fig. 2a). In December, surface waters cooled, and the mixing period started. In January, when the mixed layer temperatures dropped below $19^{\circ} \mathrm{C}$ (Fig. $3 \mathrm{a})$, nutrients were pumped to the surface, promoting a progressive increase in chlorophyll $a$ (Figs. 2, 3a). After the bloom, in April-May, the thermocline started to reform, leading to a surface euphotic zone depleted of inorganic nutrients. Environmental parameters on the eastern flank (Stations 2 and 3) were significantly different from those in the island wake (Stations 4 and 5). Stations located to the east of the island showed lower temperature and salinity values than those to the south (Friedman ANOVA, $\mathrm{p}<0.01$, in both cases). Maximum chlorophyll $a$ concentrations were measured at the southern stations located in the warm wake. 

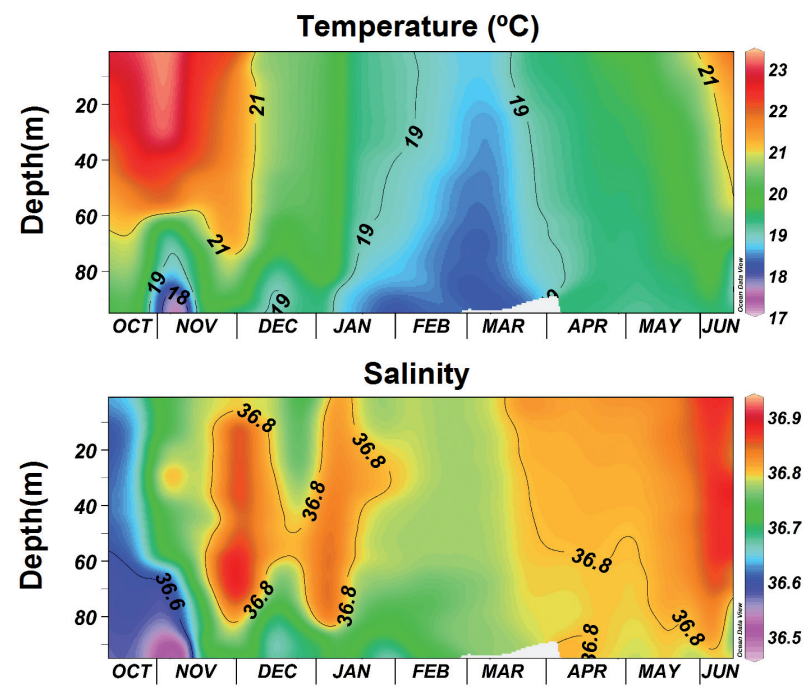

Chlorophyll a (mg Chl a.m $\left.{ }^{-3}\right)$

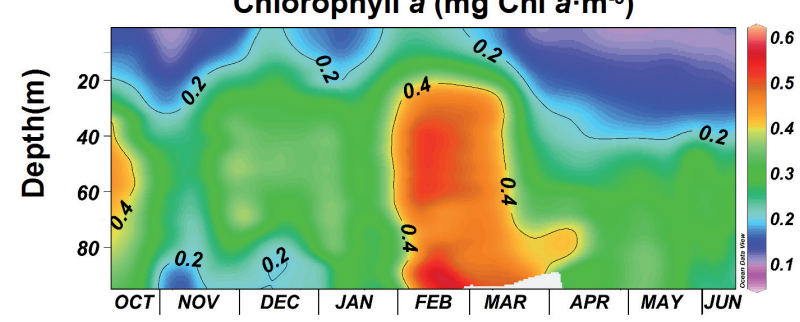

FIG. 2. - Temporal variation of a) temperature $\left({ }^{\circ} \mathrm{C}\right)$, b) salinity and c) chlorophyll $a\left(\mathrm{mg} \mathrm{Chl} a \cdot \mathrm{m}^{-3}\right)$ during the sampling period in the warm lee area (Station 5).

During the late winter bloom, chlorophyll $a$ concentrations peaked twice (Fig. 3a). The highest mesozooplankton biomass was recorded 24 days after the second peak (Fig. 3b). Mean mesozooplankton biomass was $12.3 \pm 8.9 \mathrm{SD} \mathrm{mg}$ dry weight $\cdot \mathrm{m}^{-3}$. The ichthyoplankton distribution was highly variable during the whole study period (Fig. 3c). Average concentrations were $1.05 \pm 1.4 \mathrm{SD}$ eggs $\cdot \mathrm{m}^{-3}$ and $1.2 \pm$ $0.8 \mathrm{SD}$ larvae $\cdot \mathrm{m}^{-3}$. Neritic larval abundances did not show significant differences across months (Friedman ANOVA, $\mathrm{p}>0.05$ ), whereas oceanic larvae did (Friedman ANOVA, p<0.01). Oceanic larvae were more abundant in January, February and October.

The ichthyoplankton abundance and mesozooplankton biomass values suggested a monthly periodicity (Fig. 3b,c), and therefore the lunar cycle was considered as an influencing factor (Figs. 3, 4). Lunar illumination explained the mesozooplankton biomass trend of the two size fractions, as significantly lower values were found during the new moon and higher values were found during the crescent and full moon (KW-ANOVA, $\mathrm{p}<0.005)$. However, lower abundance of neritic larvae also appeared during the new moon, although this pattern was not a)

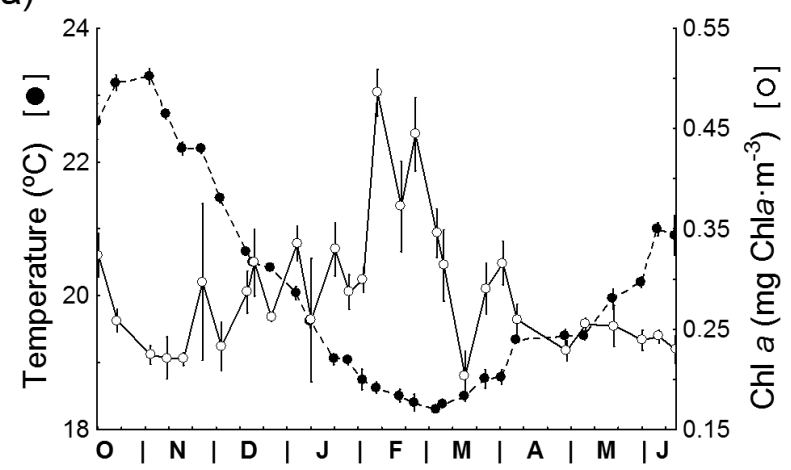

b)

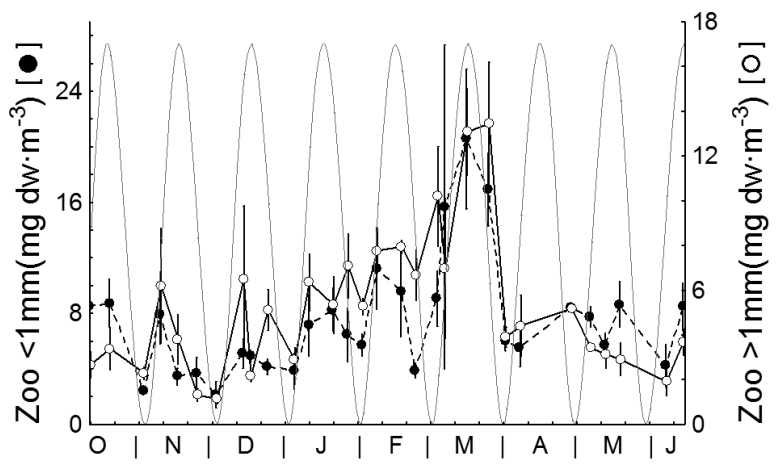

c)

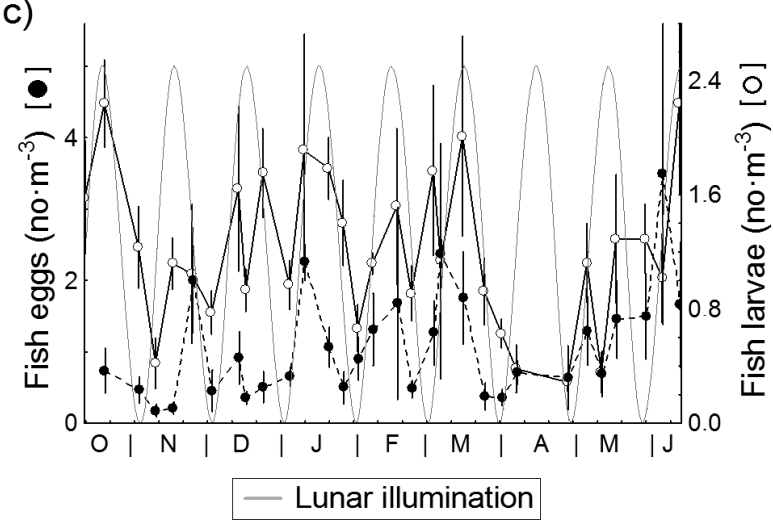

FIG. 3. - Temporal distribution of a) average temperature in the

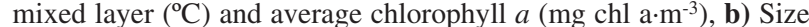
fractionated mesozooplankton biomass $\left(\mathrm{mg}\right.$ dry weight $\left.\cdot \mathrm{m}^{-3}\right)$, c) concentration of fish eggs (no.eggs $\cdot \mathrm{m}^{-3}$ ) and fish larvae $\left(\right.$ no.larvae $\cdot \mathrm{m}^{-3}$ ). Average values for the five sampling stations are shown. Note that lunar illumination is plotted as a fine grey line in the background for $3 \mathrm{~b}$ and $3 \mathrm{c}$.

significant (KW-ANOVA, $\mathrm{p}>0.05)$. Oceanic larvae did not show any significant trend (KW-ANOVA, $\mathrm{p}>0.5$ ).

The planktonic community was not homogeneously distributed along the island's shelf. Small and large-sized mesozooplankton biomass had higher values south of the island (Friedman ANOVA, $\mathrm{p}<0.05$ ). Similarly, fish eggs and neritic larvae were found in significantly lower concentrations at the eastern stations (Fig. 5; Friedman ANOVA, 
a)

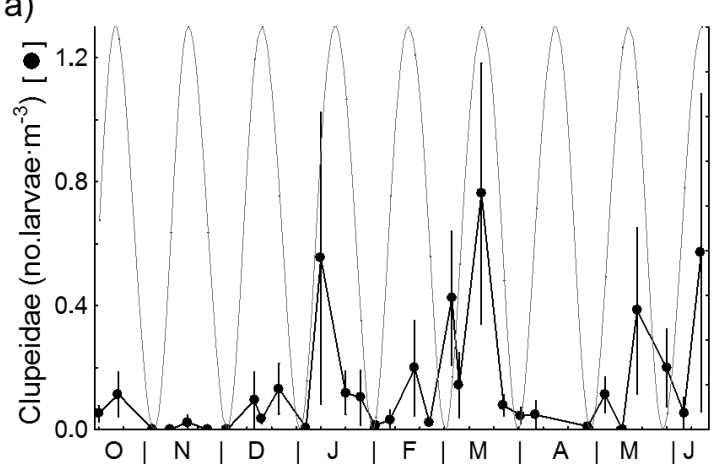

b)

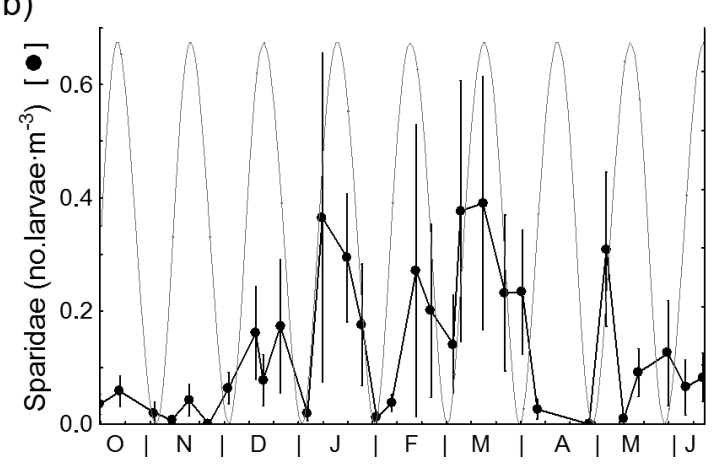

c)

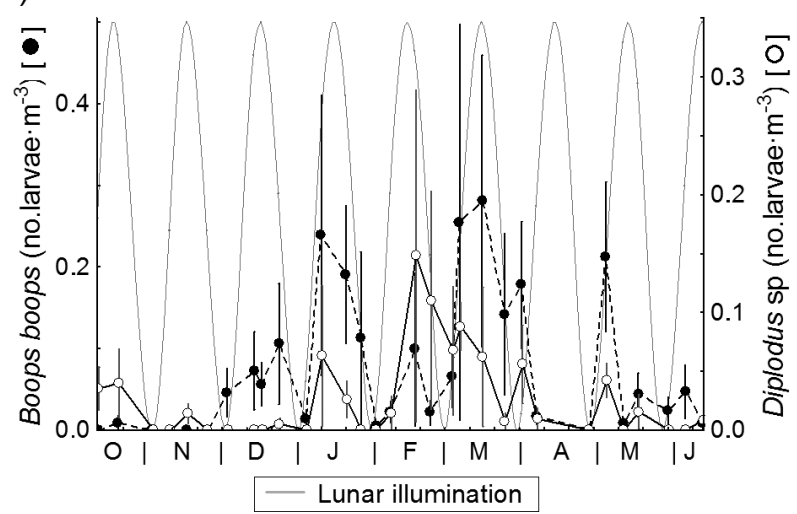

FIG. 4. - Temporal variation of average abundance (no. larvae $\cdot \mathrm{m}^{-3}$ ) of a) Clupeidae larvae, b) Sparidae larvae and c) Boops boops and Diplodus sp larvae. Note that lunar illumination is plotted in all graphs as a fine grey line. $\mathrm{p}<0.001)$. However, oceanic larval abundance did not exhibit significant differences among stations $(\mathrm{p}>0.05)$.

Environmental factors (average temperature and salinity in the mixed layer, and chlorophyll $a$ concentration) were correlated with mesozooplankton biomass (Table 1). In addition, the biomass of both size-fractions was positively correlated with fish eggs and neritic larval abundance. Oceanic larval abundance showed a different temporal trend than fish eggs and neritic larvae, and therefore they were only correlated with salinity in the mixed layer $(\mathrm{p}<0.001)$.

\section{Ichthyoplankton taxonomic composition}

A total of 3897 fish larvae, comprising 121 taxa from 41 families, were caught (Table 2). Myctophidae was by far the most abundant family (31.5\% of total larvae collected), and was mainly represented by Ceratoscopelus warmingii, Hygophum hygomii and the species of the genera Notoscopelus and Lampanyctus. The high contribution of mesopelagic species was also evidenced by the relatively high abundance of Gonostomatidae (7.8\% of total larvae), with Cyclothone braueri being the most frequent.

Sparidae, Clupeidae and Gobiidae were the families that contributed most to the neritic larvae collected $(10.4 \%, 8.7$ and $6.6 \%$ of total larvae respectively). Sardinella aurita and Boops boops were the most abundant species within this neritic group. Gobiidae were not identified at species level due to the high number of species that inhabit the region and the complexity of the identification process.

Since this study did not cover the complete annual cycle of the ichthyoplankton assemblage, a seasonal analysis could lead to biased conclusions.

TABLE 1. - Matrix showing the Spearman correlation coefficients for environmental and biotic variables. Abbreviations: Temp (Average temperature in the mixed layer, ${ }^{\circ} \mathrm{C}$ ), Salinity (Average salinity in the mixed layer), Chl $a$ (Chlorophyll $a, \mathrm{mg} \mathrm{Chl} a \cdot \mathrm{m}^{-3}$ ), Sm Zoop, Lg Zoop (Small and Large Zooplankton size fraction Biomass, mg dry weight $\cdot \mathrm{m}^{-3}$ ), F Eggs (Fish Eggs, no $\cdot \mathrm{m}^{-3}$ ), F Larvae (Fish Larvae, no $\mathrm{m}^{-3}$ ), Neritic L, Oceanic L (Neritic and Oceanic Larvae, no $\cdot \mathrm{m}^{-3}$ ).

\begin{tabular}{|c|c|c|c|c|c|c|c|c|}
\hline & Temp & Salinity & Chl $a$ & Sm Zoop & Lg Zoop & F Eggs & F Larvae & Neritic L \\
\hline Salinity & $0.37 *$ & & & & & & & \\
\hline Chl $a$ & $-0.49 *$ & $-0.38 *$ & & & & & & \\
\hline Sm. Zoop. & $-0.34 *$ & $-0.36 *$ & 0.18* & & & & & \\
\hline Lg. Zoop. & $-0.63 *$ & $-0.49 *$ & $0.29 *$ & $0.56^{*}$ & & & & \\
\hline F Eggs & -0.11 & -0.002 & 0.09 & $0.34 *$ & $0.22 * *$ & & & \\
\hline F Larvae & 0.03 & -0.12 & $0.18 * *$ & $0.34 *$ & $0.22 *$ & 0.38 & & \\
\hline Neritic L & 0.01 & 0.09 & 0.08 & $0.37 *$ & $0.21 * *$ & $0.41 *$ & $0.68 *$ & \\
\hline Oceanic L & 0.04 & $-\mathbf{0 . 3 2} *$ & 0.24 & 0.09 & 0.10 & 0.13 & $0.66 *$ & 0.10 \\
\hline
\end{tabular}


TABLE 2. - Taxonomic list of larvae collected and percentage contribution of each taxa to the total larvae collected each month and during the complete study period.

\begin{tabular}{|c|c|c|c|c|c|c|c|c|c|c|}
\hline & $\mathrm{O}$ & $\mathrm{N}$ & $\mathrm{D}$ & $\mathrm{J}$ & $\mathrm{F}$ & M & A & M & $\mathrm{J}$ & $\%$ \\
\hline $\begin{array}{l}\text { ANGUILIFORMES } \\
\text { FAMILY CLUPEIDAE }\end{array}$ & 0.73 & 0.5 & 0.14 & & & & & & & 0.16 \\
\hline Sardina pilchardus (Walbaum, 1972) & & & 0.28 & 0.16 & & 0.17 & & & & 0.10 \\
\hline Sardinella aurita (Valenciennes, 1847) & 1.21 & 0.76 & 4.50 & 6.57 & 4.46 & 17.08 & 6.85 & 6.13 & 14.01 & 6.93 \\
\hline Sardinella maderensis (Lowe, 1838) & 2.42 & & 0.14 & 0.16 & & & & 0.99 & 1.17 & 0.51 \\
\hline $\begin{array}{l}\text { Unidentified spp } \\
\text { FAMILY BATHYLAGIDAE }\end{array}$ & & & & 1.28 & & 0.66 & 1.37 & 6.52 & 0.39 & 1.21 \\
\hline Bathylagidae sp.1 & & & 1.83 & 1.44 & & 0.17 & & & & 0.59 \\
\hline FAMILY GONOSTOMATIDAE & & & & & & & & & & \\
\hline Cyclothone acclinidens (Garman, 1899) & 0.48 & & 0.14 & & & & & & & 0.08 \\
\hline Cyclothone braueri (Jespersen \& Tåning, 1926) & 9.93 & 5.81 & 5.63 & 8.17 & 7.64 & 4.98 & 2.74 & 6.13 & 8.17 & 6.75 \\
\hline $\begin{array}{l}\text { Cyclothone pallida (Brauer, 1902) } \\
\text { Cyclothone pseudopalida (Mukhacheva, 1964) }\end{array}$ & 0.73 & 0.25 & 0.14 & & & & & & & $\begin{array}{l}0.03 \\
0.10\end{array}$ \\
\hline $\begin{array}{l}\text { Cyclothone pseudopallad (Muknacneva, 1904) } \\
\text { Cyclothone sp. }\end{array}$ & $\begin{array}{l}0.13 \\
2.18\end{array}$ & $\begin{array}{l}0.25 \\
1.26\end{array}$ & 0.28 & 0.32 & & & & & & 0.46 \\
\hline Gonostoma atlanticus (Norman, 1930) & & & & & & 0.17 & & & & 0.03 \\
\hline Gonostoma spp & 0.24 & & & & & & & & & 0.03 \\
\hline Unidentified spp & & 1.01 & 0.14 & 0.16 & 0.32 & & 5.48 & 0.20 & & 0.31 \\
\hline $\begin{array}{l}\text { FAMILY STERNOPTYCHIDAE } \\
\text { Argyropelecus hemigymnus (Cocco,1829) }\end{array}$ & & & & & & & & & & \\
\hline $\begin{array}{l}\text { Argyropelecus hemigymnus (Cocco,1829) } \\
\text { FAMILY STOMIIDAE }\end{array}$ & 0.24 & 0.25 & & 0.16 & 0.96 & 0.33 & & & & 0.21 \\
\hline $\begin{array}{l}\text { FAMILY STOMIIDAE } \\
\text { Idiacanthus fasciola (Peters, 1877) }\end{array}$ & & & 0.14 & & & & & & & 0.03 \\
\hline Stomias boa (Risso, 1810) & & & 1.97 & 0.80 & 0.32 & 0.66 & & 0.20 & & 0.64 \\
\hline Unidentified spp & & & 0.28 & 0.48 & 0.96 & 0.17 & & & & 0.23 \\
\hline FAMILY PHOTICHTHYiDAE & & & & & & & & & & \\
\hline Ichthyococcus ovatus $($ Cocco, 1938) & & & & & 0.64 & 0.17 & & & & 0.08 \\
\hline Vinciguerria attenuata (Соссо, 1938) & 0.24 & & & & & & & & & 0.03 \\
\hline Vinciguerria nimbaria (Jordan \& Williams, 1896) & 0.97 & 0.51 & & 0.32 & 0.32 & 0.17 & & 0.59 & & 0.33 \\
\hline Vinciguerria poweriae (Cocco, 1938) & 0.48 & 0.25 & 0.70 & 0.80 & 0.96 & 0.17 & 1.37 & 0.59 & 0.39 & 0.56 \\
\hline Vinciguerria spp & 0.97 & 0.25 & 0.28 & 0.64 & 6.37 & 1.82 & & 0.40 & 1.56 & 1.23 \\
\hline FAMILY MALACOSTEIDAE & & & & & & & & 0.20 & & 0.03 \\
\hline $\begin{array}{l}\text { FAMILY CHLOROPHTHALMIDAE } \\
\text { Chlorophthalmus sp.1 }\end{array}$ & & & & & & & & 0.20 & & 0.03 \\
\hline $\begin{array}{l}\text { Chlorophthalmus sp.. } \\
\text { FAMILY NOTOSUDIDAE }\end{array}$ & & & & & & & & 0.20 & & 0.05 \\
\hline Scopelosaurus lepidus (Krefft \& Maul, 1955) & & & & & 0.32 & 0.17 & & & & 0.05 \\
\hline $\begin{array}{l}\text { FAMILY SYNODONTIDAE } \\
\text { Synodontidae sp.1 }\end{array}$ & & & & & & & & & & \\
\hline $\begin{array}{l}\text { Synodontidae sp.1 } \\
\text { FAMILY MYCTOPHIDAE }\end{array}$ & & 0.76 & 0.28 & 0.32 & 0.32 & & 1.37 & & 0.39 & 0.26 \\
\hline Ceratoscopelus maderensis (Lowe, 1839) & 0.24 & 1.01 & 0.84 & & & 0.17 & 2.74 & 0.40 & 0.39 & 0.44 \\
\hline Ceratoscopelus warmingii (Lütken, 1892) & 7.75 & 4.29 & 4.36 & 1.76 & & 0.17 & 5.48 & 10.67 & 5.45 & 4.21 \\
\hline Diaphus brachycephalus (Taning, 1928) & & & & & & & & & 0.39 & 0.03 \\
\hline Diaphus holti (Taning, 1918) & 0.73 & & & & & & & & & 0.08 \\
\hline Diaphus metopoclampum (Cocco, 1829) & 0.24 & & & & & & & 0.20 & & 0.05 \\
\hline Diaphus mollis (Taning, 1928) & & 0.25 & & 0.16 & & & & 0.40 & 0.78 & 0.15 \\
\hline Diaphus rafinesquii $($ Cocco, 1838) & 0.48 & 0.51 & 0.56 & 0.32 & & 0.17 & & 0.20 & & 0.31 \\
\hline Diaphus spp & 2.18 & 0.76 & 0.98 & 0.96 & 0.32 & & & 1.58 & 0.78 & 0.92 \\
\hline Diogenichthys atlanticus (Tåning, 1918) & 0.97 & 0.76 & 0.42 & 0.16 & 1.91 & 1.16 & & 0.20 & & 0.64 \\
\hline Hygophum benoiti (Cocco, 1838) & 1.21 & 0.25 & & 0.48 & 1.27 & 0.17 & & & & 0.36 \\
\hline Hygophum hygomii (Lütken, 1892) & 3.87 & 7.07 & 8.72 & 3.04 & 0.32 & 0.50 & & 0.20 & & 3.34 \\
\hline Hygophum macrochir (Günther, 1864) & 1.45 & 0.76 & 1.55 & 0.80 & 0.32 & & & 0.20 & 0.39 & 0.72 \\
\hline Hygophum reinhardtii (L & 1.45 & 4.04 & 1.13 & 0.32 & & 0.17 & & 0.20 & 0.39 & 0.90 \\
\hline Hygophum taaningi (Becker, 1965) & 0.97 & 0.76 & 0.98 & 1.12 & 0.32 & 0.33 & & 0.20 & 0.78 & 0.69 \\
\hline Hygophum spp & 1.94 & 0.76 & 0.84 & 1.28 & 0.64 & 0.50 & & 0.40 & & 0.82 \\
\hline Lampadena sp.1 & & & & 0.48 & & & & & 0.39 & 0.10 \\
\hline Lampadena spp & 2.66 & 1.26 & 0.28 & 0.16 & 0.32 & & 1.37 & 1.38 & & 0.72 \\
\hline yctus sp.1 & 0.97 & 0.25 & & & & & & & & 0.13 \\
\hline Lampanyctus spp & 4.84 & 4.04 & 2.53 & 2.88 & 4.14 & 2.49 & 8.22 & 1.58 & 2.33 & 3.08 \\
\hline Lepidophanes guentheri (Goode \& Bean, 1896) & & 0.25 & 0.14 & & 0.64 & & & & & 0.10 \\
\hline Lobianchia dofleini (Zugmayer, 1911) & & & 2.11 & 4.65 & 1.27 & 1.82 & 1.37 & & 0.39 & 1.57 \\
\hline Lobianchia gemellarii (Cocco, 1938) & & & 1.69 & 1.44 & 0.32 & 0.50 & & & & 0.64 \\
\hline Lobianchia spp & 0.48 & 2.27 & 3.09 & 2.72 & 1.59 & 1.66 & & 0.40 & & 1.72 \\
\hline Myct & 0.24 & & & & & & & 0.20 & & 0.05 \\
\hline Myctophidae sp. 2 & 2.91 & 1.77 & 0.70 & & & & & & 0.39 & 0.64 \\
\hline idae sp.3 & 1.94 & 0.25 & & 0.48 & & & & & & 0.31 \\
\hline Myctophidae sp. 4 & & & & 0.32 & 0.32 & 1.00 & 4.11 & 0.20 & 0.39 & 0.36 \\
\hline Myctophum nitidulum (Garman, 1899) & 2.18 & 0.51 & 0.70 & 0.32 & 0.64 & 0.17 & & & & 0.54 \\
\hline Myctophum selenops (Tåning, 1928) & & & 0.28 & & 0.64 & & & & & 0.10 \\
\hline Nannobrachium lineatum (Tăning, 1928) & & & & & & & & & 0.78 & 0.05 \\
\hline Notolychnus valdiviae (Brauer, 1904) & & 0.76 & & & & & & & & 0.08 \\
\hline Notoscopelus resplendens (Richardson, 1845) & 0.24 & & 0.28 & 0.80 & 0.64 & 0.50 & & 0.40 & & 0.38 \\
\hline Notoscopelus spp & 0.97 & 0.51 & 2.39 & 5.77 & 6.37 & 3.98 & 2.74 & 1.19 & 3.89 & 3.10 \\
\hline Symbolophorus spp & & & 0.28 & 0.48 & 0.32 & 0.50 & & & & 0.23 \\
\hline Unidentified spp & 4.60 & 5.05 & 4.50 & 5.13 & 6.05 & 2.99 & 5.48 & 0.59 & 2.72 & 3.95 \\
\hline FAMILY EVERMANELLIDAE & & & 0.28 & 0.16 & & & & & & 0.08 \\
\hline FAMILY PARALEPIDAE & & & & & & & & & & \\
\hline Lestidiops jakari pseudospyraenoides (Ege, 1918) & & 0.25 & & 0.32 & & & & & & 0.08 \\
\hline Sudis hyalina (Rafinesque, 1810) & & & & 0.16 & & & & & & 0.03 \\
\hline Unidentified spp & & & 0.14 & & & & & 0.20 & 0.39 & 0.08 \\
\hline
\end{tabular}




\begin{tabular}{|c|c|c|c|c|c|c|c|c|c|c|}
\hline & $\mathrm{O}$ & $\mathrm{N}$ & $\mathrm{D}$ & $\mathrm{J}$ & $\mathrm{F}$ & M & A & M & $\mathrm{J}$ & $\%$ \\
\hline $\begin{array}{l}\text { FAMIILY MACRORHAMPHOSIDAE } \\
\text { Macroramphosus scolopax (Linnaeus, 1758) }\end{array}$ & 0.48 & 0.51 & 0.14 & & 1.27 & 0.50 & & & & 0.31 \\
\hline $\begin{array}{l}\text { FAMILY SYGNATHIDAE } \\
\text { Hippocampus hippocampus (Linnaeus, 1758) }\end{array}$ & & & & & & & & & 0.39 & 0.03 \\
\hline FAMILY MELAMPHAIDAE & & & & & & & & & & \\
\hline $\begin{array}{l}\text { Melamphaes simus (Ebeling, 1962) } \\
\text { FAMILY GADIDAE }\end{array}$ & & 0.25 & 0.42 & 0.16 & & & & & & 0.13 \\
\hline Phycis spp & & & & & 0.32 & & & & & 0.03 \\
\hline $\begin{array}{l}\text { FAMILY HEMIRHAMPHIDAE } \\
\text { Hemirhamphus balao (Le Sueur, 1823) }\end{array}$ & 0.58 & & & & & 0.17 & & & & 0.03 \\
\hline FAMILY CAPROIDAE & & & & & & & & & & \\
\hline $\begin{array}{l}\text { Capros aper (Linnaeus, 1758) } \\
\text { FAMILY SERRANIDAE }\end{array}$ & & & 0.14 & 1.28 & 0.64 & & & & & 0.28 \\
\hline Anthias anthias (Linnaeus, 1758) & 0.48 & 0.25 & 1.13 & 0.16 & 0.64 & 0.17 & 1.37 & 1.38 & 3.50 & 0.82 \\
\hline $\begin{array}{l}\text { Serranidae sp.1 } \\
\text { Serranus cabrilla (Linnaeus, 1758) }\end{array}$ & & & 0.14 & 0.16 & 0.32 & 0.33 & & 0.59 & 0.39 & $\begin{array}{l}0.10 \\
0.13\end{array}$ \\
\hline Serranus spp & & & 0.17 & 0.32 & & & & 0.20 & 0.07 & 0.08 \\
\hline Unidentified spp & 0.24 & & 0.14 & 0.48 & & 0.17 & & 0.79 & 0.39 & 0.28 \\
\hline FAMILY CARANGIDAE & & & & & & & & & & \\
\hline $\begin{array}{l}\text { Trachurus picturatus (Bowdich, 1825) } \\
\text { Unidentified spp }\end{array}$ & & & & 0.32 & 0.64 & $\begin{array}{l}0.33 \\
0.17\end{array}$ & & 0.20 & & $\begin{array}{l}0.18 \\
0.03\end{array}$ \\
\hline FAMILY CORYPHAENIDAE & & & & & & & & & & \\
\hline $\begin{array}{l}\text { Coryphaena hippurus (Linnaeus, 1758) } \\
\text { FAMILY MULLIDAE }\end{array}$ & & & 0.28 & & 0.32 & 0.83 & 1.37 & & 0.39 & 0.26 \\
\hline Mullus barbatus (Linnaeus, 1758) & & & & & & & & 0.59 & & 0.08 \\
\hline FAMILY BRAMIDAE & 0.48 & & 0.14 & & & 0.66 & & & & 0.18 \\
\hline FAMILY SPARIDAE & & & & & & & & & & \\
\hline Boops boops (Linnaeus, 1758) & 0.24 & & 5.91 & 6.89 & 3.18 & 12.94 & 2.74 & 9.49 & 1.56 & 5.85 \\
\hline Diplodus spp & 1.94 & 0.51 & 0.14 & 0.80 & 8.92 & 4.31 & 1.37 & 1.78 & 0.39 & 2.08 \\
\hline Oblada melanura (Linnaeus, 1758) & & 0.25 & & & 3.82 & 1.49 & & 2.17 & & 0.85 \\
\hline Pagellus acarne? (Risso, 1826) & & 0.76 & 1.27 & 0.32 & & 0.50 & & & & 0.44 \\
\hline Pagellus bogaraveo (Brünnich, 1768) & & & 0.98 & & 0.32 & & & & & 0.21 \\
\hline Pagellus spp & & & & & 0.32 & & & & & 0.03 \\
\hline Pagrus pagrus (Linnaeus, 1758) & 0.24 & & & 0.32 & & & & 0.40 & 1.17 & 0.21 \\
\hline Unidentified spp & & 0.76 & 1.27 & 1.76 & & 0.17 & & 0.99 & 0.78 & 0.80 \\
\hline FAMILY CENTRACANTHIDAE & & & & & & & & & & \\
\hline Centracanthus cirrus (Rafinesque, 1810) & 0.24 & & 0.28 & 0.32 & 0.64 & & & & & 0.18 \\
\hline $\begin{array}{l}\text { Unidentified spp } \\
\text { FAMILY LABRIDAE }\end{array}$ & & & 0.14 & 0.16 & & & & & & 0.05 \\
\hline FAMILY LABRIDAE & & & & & & & & & & \\
\hline $\begin{array}{l}\text { Coris julis (Linnaeus, 1758) } \\
\text { Symphodus spp }\end{array}$ & 0.48 & & & & 0.32 & $\begin{array}{l}0.17 \\
0.50\end{array}$ & & 0.20 & 0.39 & $\begin{array}{l}0.10 \\
0.13\end{array}$ \\
\hline Thalassoma pavo (Linnaeus, 1758) & & & & 0.16 & & & & 0.20 & & 0.05 \\
\hline Unidentified spp & & 0.51 & 0.14 & 0.16 & 0.32 & 0.33 & & 0.40 & 0.39 & 0.26 \\
\hline FAMILY POMACENTRIDAE & & & & & & & & & & \\
\hline Pomacentridae sp.1 & 0.24 & & 2.81 & 1.76 & 1.59 & 0.50 & 2.74 & 0.40 & & 1.13 \\
\hline Pomacentridae sp.2 & 0.24 & & & & & & & 0.99 & 8.56 & 0.72 \\
\hline Unidentified spp & & & & 0.32 & & & & & & 0.05 \\
\hline FAMILY CHIASMODONTIDAE & & & & & & & & & & \\
\hline $\begin{array}{l}\text { Chiasmodon níger } \\
\text { FAMILY TRACHINIDAE }\end{array}$ & & & 0.14 & 0.16 & & & & & & 0.05 \\
\hline Trachinus draco (Linnaeus, 1758) & 0.97 & 0.25 & & & & & & & & 0.13 \\
\hline FAMILY GEMPYLIDAE & & & & & & & & & & \\
\hline $\begin{array}{l}\text { Diplospinus multistriatus (Maul, 1948) } \\
\text { Nealotus tripes (Jonhson. 1865) }\end{array}$ & & & $\begin{array}{l}0.70 \\
0.14\end{array}$ & 0.96 & & 0.33 & & & & $\begin{array}{l}0.33 \\
0.03\end{array}$ \\
\hline Unidentified spp & & 0.51 & 0.14 & & & & & & 0.78 & 0.13 \\
\hline FAMILY TRICHIURIDAE & & & & & & & & & & \\
\hline Benthodesmus elongatus (Clarke, 1879) & & 0.25 & 0.98 & 0.32 & 0.64 & & & & & 0.31 \\
\hline FAMILY SCOMBRIDAE & & & & & & & & & & \\
\hline Sarda sarda (Bloch, 1793) & & & & & 0.32 & & & & & 0.03 \\
\hline $\begin{array}{l}\text { Scomber colias (Houttuyn, 1792) } \\
\text { Unidentified spp }\end{array}$ & & & & 2.72 & 0.96 & 0.66 & & & 1.17 & 0.69 \\
\hline FAMILY GOBIIDAE & & & & & & & & & & \\
\hline Lebetus guilletii (Le Danois, 1913) & & & & & 0.64 & & & 0.20 & & 0.08 \\
\hline Unidentified spp & 5.57 & 30.81 & 2.53 & 1.28 & 2.23 & 5.64 & 9.59 & 2.77 & 8.17 & 6.52 \\
\hline FAMILY BLENNIIDAE & & & & & & & & & & \\
\hline Ophioblennius atlanticus (Valenciennes, 1836) & 0.73 & & & 0.16 & 0.32 & 0.17 & & 1.38 & 1.17 & 0.41 \\
\hline FAMILY OPHIDIIDAE & & & & & & & & 0.40 & 0.78 & 0.10 \\
\hline FAMILY TETRAGONURIDAE & & & & & & & & & & \\
\hline $\begin{array}{l}\text { Tetragonurus atlanticus (Lowe, 1839) } \\
\text { FAMILY SCORPAENIDAE }\end{array}$ & & & 0.42 & 0.80 & & 0.17 & & & & 0.23 \\
\hline $\begin{array}{l}\text { FAMILY SCORPAENIDAE } \\
\text { Scorpaena porcus (Linnaeus, 1758) }\end{array}$ & & & & & & & & & & \\
\hline $\begin{array}{l}\text { Scorpaena porcus (Linnaeus, 1758) } \\
\text { Scorpaenidae Pte. } 1\end{array}$ & & & 0.14 & $\begin{array}{l}0.16 \\
0.32\end{array}$ & & 0.33 & & & & $\begin{array}{l}0.10 \\
0.05\end{array}$ \\
\hline Unidentified spp & & & 1.13 & 1.12 & 0.96 & 1.00 & & & 0.39 & 0.64 \\
\hline FAMILY TRIGLIDAE & & & 0.14 & 0.32 & & 0.17 & & & & 0.10 \\
\hline FAMILY BOTHIDAE & & & & & & & & & & \\
\hline Arnoglossus imperialis (Rafinesque, 1819) & & & & 0.16 & & & & & & 0.03 \\
\hline Arnoglossus thori (Kyle, 1913) & 0.48 & 0.51 & 0.14 & 0.16 & & & & & & 0.15 \\
\hline Arnoglossus spp & & 0.25 & 0.28 & & & & & & & 0.08 \\
\hline Bothus podas (Delaroche, 1809) & 0.48 & & & & & & & & & 0.05 \\
\hline Unidentified spp & & & 0.42 & 0.48 & & & & & 0.39 & 0.18 \\
\hline FAMILY TETRAODONTIDAE & 0.48 & & 0.14 & & & & & & 1.17 & 0.15 \\
\hline
\end{tabular}




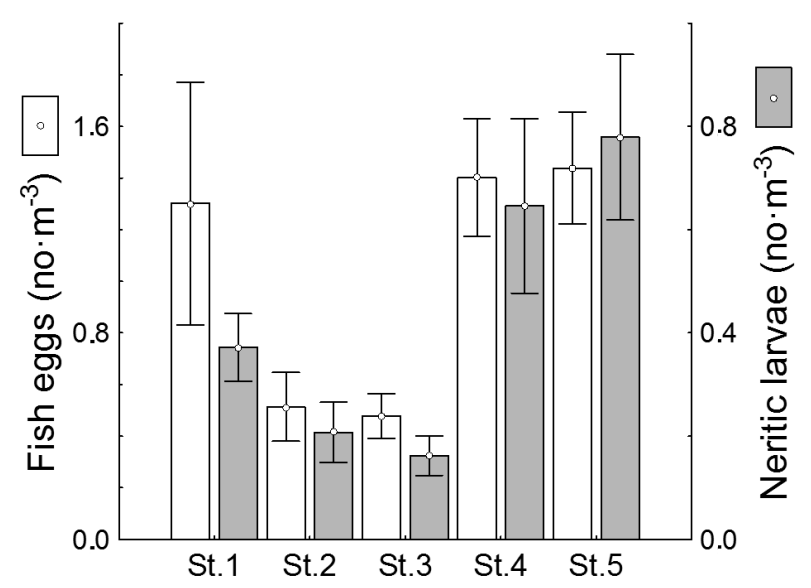

FIG. 5. - Average abundance (no. $\mathrm{m}^{-3}$ ) and standard error (SE) along the five sampling stations of total fish eggs (white bars) and neritic larvae (grey bars).

However, some strong trends were observed in the spawning patterns of some species. During winter, Boops boops, Sardinella aurita and Cyclothone braueri dominated the larval assemblage (Table 2). Other less abundant species, such as Trachurus picturatus and Scomber colias, considered winter spawners in the region, were only found during this period. On the other hand, warm water species such as Ceratoscopelus warmingii and Hygophum hygomii appeared in higher densities during autumn and late spring. Pomacentridae sp2 and Trachinus draco are probably summer spawners in the region, as they were only found during the stratified period.

The temporal distributions of the families Clupeidae and Sparidae were analyzed in detail due to their relatively high abundances and their important ecological and commercial roles (Fig. 4). Both families displayed a monthly trend similar to that shown by mesozooplankton biomass (Fig. 4a,b). Sparidae larvae exhibited significantly lower abundances during the new moon (KW-ANOVA, $\mathrm{p}<0.05)$ and Boops boops showed higher density peaks during the illuminated phase of the lunar cycle (i.e., crescent and full moon) (KW-ANOVA, p<0.05) (Fig. 4). Larvae of the genus Diplodus also showed lower values during the new moon, although these differences were not significant (KW-ANOVA, p>0.005) (Fig. 4c).

ANOSIM results indicated that there was a significant difference between months (seasonality), whereas sampling sites only seemed to have a marginal effect on the community composition during the sampling period (ANOSIM, $\mathrm{R}=0.275, \mathrm{p}<0.01$ and $\mathrm{R}=0.055, \mathrm{p}<0.05$ respectively). Although sampling site did not significantly affect the composition

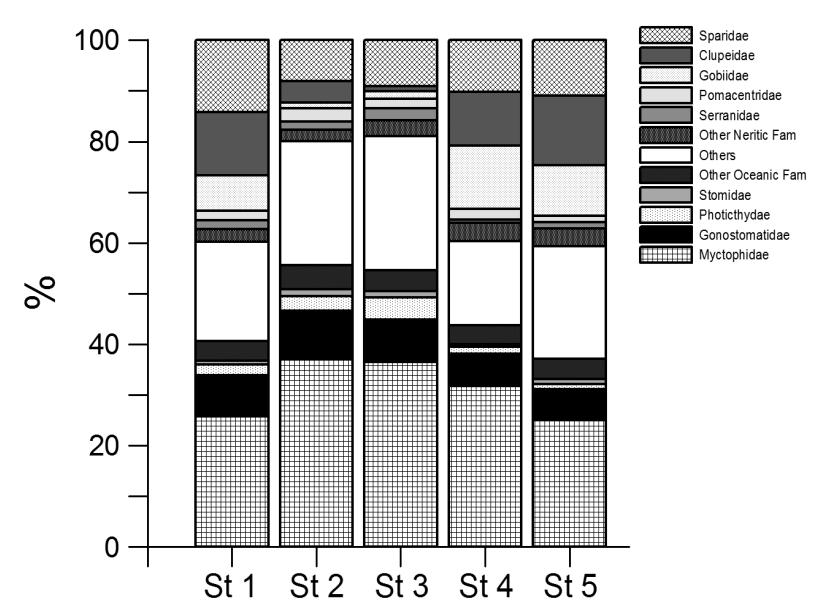

FIG. 6. - Percentage contribution of the nine most abundant families (contributing $>0.9 \%$ of total collected larvae) to the total larval fish catches at each sampling station.

and structure of the complete larval fish assemblage, two neritic families, Clupeidae and Gobiidae, were found in significantly higher abundances in the retention spots upstream (Station 1) and downstream (Stations 4 and 5) of the island (Fig. 6, Friedman ANOVA $\mathrm{p}<0.01)$. However, mesopelagic families (e.g. Myctophidae, Gonostomatidae, and Phosichthyidae) had slightly higher abundances on the eastern flank of the island where the flow is locally enhanced, although this pattern was not significant (Friedman ANOVA $\mathrm{p}>0.05$ ).

\section{DISCUSSION}

A typical feature of subtropical waters is that seasonal patterns are weak (Barton et al., 1998). The water column is strongly stratified almost all year round, showing a seasonal thermocline and a deep chlorophyll maximum (Hernández-León et al., 2007). Trade Winds reach maximum strength in summer and, even so, maximum temperatures are recorded during this stratified period (Barton et al., 1998). Surface cooling during winter combined with wind stirring weakens the almost permanent stratification. This short mixing stage promotes the most productive season in these waters, although its magnitude and extension are much smaller than in temperate waters (Arístegui et al., 2001). These authors attributed the first chlorophyll peak to an abrupt increase in phytoplankton cells $<2 \mu \mathrm{m}$ (mostly diatoms), whereas in the second peak, there were higher densities of cells $>2 \mu \mathrm{m}$. In our study, maximum abundances of mesozooplankton biomass were 
registered with a lag of three weeks after the second chlorophyll peak. This mesozooplankton bloom was split into two peaks, which has been related to the result of the interplay between resource and consumer controls (Hernández-León et al., 2004). These authors considered that although resources were a limiting factor after the first zooplankton bloom, mesozooplankton biomass variability was a consequence of the predatory pressure exerted by diel vertical migrants.

Average values of mesozooplankton biomass and larval fish abundance were found in the expected range of previous studies (Rodríguez et al., 2000; Hernández-León et al., 2004; Becognée et al., 2006; Moyano et al., 2009). Larval fish diversity was high, which is typical of subtropical waters (Longhurst and Pauly, 1987). The high contribution of mesopelagic species (i.e., Myctophids and Gonostomatids) is characteristic of oceanic islands due to the narrow island shelf. Therefore, the composition of the larval fish community, at the family-level, is similar to that of other oceanic islands (Leis, 1991; Boehlert et al., 1992). Larval abundances were also similar to those found in similar studies off the Balearic Islands in the Mediterranean Sea (Alemany et al., 2006), off Australia (Smith and Suthers, 1999; Muhling et al., 2008) and in the Agulhas Current (Beckley and van Ballegooyen, 1992).

Unlike mesozooplankton, larval abundance did not show a temporal peak during the late winter bloom period. This temporal pattern of abundance may be a consequence of the low seasonality in the water properties found in the Canary Islands compared to temperate seas. This mild seasonality results in an extension of the spawning periods of fish species (Lowe-McConnell, 1987). This is the case for Gran Canaria where larvae of the most abundant species are present for several months (i.e., Boops boops) or all year round (i.e., Sardinella aurita, $C y$ clothone braueri).

Nevertheless, the larval concentrations of some species exhibited some sort of seasonality, suggesting the presence of winter and summer assemblages. During winter, the larval assemblage was dominated by Boops boops and Sardinella aurita and also characterized by the presence of Scomber colias and Trachurus picturatus. This is in agreement with the spawning periods of these species in the $\mathrm{Ca}$ nary waters. Boops boops spawns from November to July, with a maximum peak during March and April (Franquet and Brito, 1995), and in our study larvae of this species appeared in higher densities in March and also in January. Sardinella aurita spawns throughout the year around Gran Canaria, peaking in summer (June to September) and winter (December to February) (Bécognée et al., 2006). Moyano et al. (2009) also found high densities in spring. Scomber colias and Trachurus picturatus larvae appeared during the mixing period of the water column (i.e., January to March), which coincides with their spawning time described for the area (Franquet and Brito, 1995; Lorenzo and Pajuelo, 1996). Our study is the first to record early stages of both species near Gran Canaria, although Scomber colias is the most abundant pelagic species in the archipelago (Lorenzo and Pajuelo, 1996). The presence of small sized Scomber colias larvae confirms that this species reproduces successfully in the island waters, and that the larval transport within upwelling filaments from the African coast, if real, might act as a complementary source of individuals to the existing stock. Therefore, the extension of the spawning periods (Lowe-McConnell, 1987) in the area is evident when compared to temperate Mediterranean Sea waters. Both ecosystems have neritic and oceanic species in their larval community structure (Sabatés et al., 1990; Somarakis et al., 2002; Isari et al., 2008), but their presence during the year is completely different. Sardinella aurita and Hygophum hygomii are summer spawners in the Mediterranean Sea (Goodyear et al., 1972; Olivar and Palomera, 1994; Sabatés et al., 2006), while their larvae are present during the whole year off Gran Canaria (Bécognée et al., 2006). Similarly, Scomber colias is a summer spawner in the Mediterranean (Sabatés, 1990), but it only reproduces during winter in the Canaries (Lorenzo and Pajuelo, 1996).

During late summer, the larval community is dominated by the myctophids Ceratoscopelus warmingii and Hygophum hygomii. The former has a broad tropical distribution occurring worldwide (Bekker, 1983). Little is known about their reproduction in the region. Ceratoscopelus warmingii spawning peaks in spring near Hawaii (Clarke, 1973) and its larvae provide a substantial year-round contribution to the larval fish assemblage of the North Pacific Central Gyre (Loeb, 1980). Both temporal patterns in the Pacific are consistent with our results that suggest that they reproduce the whole year round, showing lower densities during the colder months. Hygophum hygomii seemed to be present in Gran Canaria waters year-round, peaking in the warmer months, as in the 
Mediterranean (Goodyear et al., 1972). Therefore, it might be reasonable to consider that they extend this period to early winter due to the warmer conditions of the Canary Island waters. In addition, Trachinus draco, Pomacentridae sp2 and Tetraodontidae species seem to be summer spawners, but little is known about their ecology in the area. Hence, these species may be characteristic of the summer assemblage, but the limited representation of the summer months during this study precludes any conclusion.

In relation to local hydrography, mesozooplankton biomass and fish eggs and neritic larval abundances benefit from local retention, showing lower values at the eastern flank of the island, where enhanced flow advects them away, and accumulating in the windward and leeward zones. This variability in the plankton community around islands has been observed in several studies (Hammer and Hauri, 1981; Boehlert and Mundy, 1993), and it has already been proposed for Gran Canaria by Rodríguez et al. (2001). Here, we confirm this pattern on a finer scale, particularly for members of the families Clupeidae and Gobiidae. However, the complete larval community composition and structure did not vary between retention and exposed sites on a long-term basis.

\section{Lunar illumination}

Probably the most striking results in this work are those related to the lunar illumination. We found clear evidence of the mesozooplankton biomass increasing during the illuminated phase of the lunar cycle: low values during the new moon that increase through the crescent and full moon, and then decrease again during the waning moon. This pattern was explained by Hernández-León (1998), for zooplankton, to be a result of predator pressure. During the full moon, diel vertical migrants (DVMs) do not reach the upper layers of the ocean $(<100 \mathrm{~m}$ depth), reducing the predatory pressure on epipelagic zooplankton. However, during the dark phase of the lunar cycle, DVMs reach the upper layers feeding on the increased zooplankton biomass. Hernández-León et al. (2001) found strong differences between the full and new moon and emphasized the importance of this process on the flux of energy and organic matter from the euphotic to the mesopelagic zone. Recently, Hernández-León (2008) formulated a hypothesis about the influence of lunar illumination on fish spawning and larval fish abundance. This author found evidence of a coupling of fish reproduction and the epipelagic zooplankton bloom during the illuminated period of the lunar cycle, and argued that this pattern may constitute a short-term version of the match-mismatch hypothesis (Cushing, 1990). This coupling would be explained as a result of (1) the lower predatory pressure on fish larvae, (2) the enhanced feeding of adult fish promoted by the increase in zooplankton during the lunar cycle, insofar as this energy intake would be converted into reproduction products, and (3) the enhanced feeding by fish larvae on zooplankton during the lunar cycle. Therefore, our results partially confirm this hypothesis for neritic larvae. Clupeids and Sparids were the clearest cases, probably because their abundances were high enough for the trend to be noticed. Maximum abundances for both groups recorded during the illuminated phase supports the idea that spawning is coupled to promote larval survival in a low-predation, high-feeding scenario. Further research on this lunar influence would provide insights into the short-term variations of the ichtyoplankton community and the natural variability of fisheries.

In conclusion, the variability of the larval fish community off Gran Canaria suggests the presence of two seasonal assemblages corresponding to the mixing and stratification periods. Despite the presence of some species year-round, winter and summer spawners seem to define the annual larval fish assemblage. In this sense, temporal variations seemed to play a more important role in the community structure than location around the island over time. The periodicity in the temporal distribution of neritic larval fish abundance and small mesozooplankton biomass questions the ultimate factors governing the larval dynamics. Finally, the influence of the DVM lunarlinked predator pressure cycle on mesozooplankton and larval fish abundances seems to govern their temporal variability. Therefore, a better understanding of larval dynamics would profoundly improve the scope of fisheries management and, especially, of marine reserves in the Archipelago.

\section{ACKNOWLEDGEMENTS}

The authors would like to thank C. Schmoker and L. Nieves for the chlorophyll data, and all the colleagues of the Biological Oceanography Group who contributed to the hard field work. Three anonymous reviewers are thanked for valuable com- 
ments that helped to improve the manuscript. This study was supported by the project ConAfrica (Cicyt CTM2004-02319) and by a FPU fellowship to M.M. (AP 2005-4742), both from the Spanish Ministry of Education and Science.

\section{REFERENCES}

Alemany, F., S. Deudero, B. Morales-Nin, J.L. López-Jurado, J. Jansà, M. Palmer and I. Palomera. - 2006. Influence of the physical environmental factors on the composition and horizontal distribution of summer larval fish assemblages off Mallorca Island (Balearic archipielago, western Mediterranean). $J$. Plankton Res., 28: 473-487.

Arístegui, J., S. Hernández-León, M.F. Montero and M. Gómez. - 2001. The seasonal planktonic cycle in coastal waters of the Canary Islands. Sci. Mar., 65: 51-58.

Barton, E.D., J. Arístegui, P. Tett, M. Cantón, J. García-Braun, S. Hernández-León, L. Nykjaer, C. Almeida, J. Almunia, S. Ballesteros, G. Basterretxea, J. Escánez, L. García-Weill, A. Hernández-Guerra, F. López-Laatzen, R. Molina, M.F. Montero, E. Navarro-Pérez, J.M. Rodríguez, K. van Lenning, H. Vélez and K. Wild. - 1998. The transition zone of the Canary Current upwelling region. Progr. Oceanogr., 41: 455-504.

Beckley, L.E. and R.C. van Ballegooyen. - 1992. Oceanographic conditions during three ichthyoplankton surveys of the Agulhas Current in 1990/91. S. Afr. J. Mar. Sci., 12: 83-93.

Bécognée, P., C. Almeida, A. Barrera, A. Hernández-Guerra and S. Hernández-León. - 2006. Annual cycle of clupeiform larvae around Gran Canaria Island, Canary Islands. Fish. Oceanogr. 15: 293-300

Bekker, V.E. - 1983. Myctophid fishes of the world ocean. Nauka Press, Moscow.

Boehlert, G.W., W. Watson and L.C. Sun. - 1992. Horizontal and vertical distributions of larval fishes around an isolated oceanic island in the tropical Pacific. Deep Sea Res. I., 39(3/4): 439-466.

Boehlert, G.W. and B.C. Mundy. - 1993. Ichthyoplankton assemblages at seamounts and oceanic islands. Bull. Mar. Sci., 53(2): 336-361.

Braun, J.G. - 1980. Estudios de producción en aguas de las Islas Canarias I. Hidrografía, nutrientes y producción primaria. Bol. Inst. Esp. Oceanogr., 5: 147-154.

Clarke, K.R. and R.M. Warwick. - 2005. PRIMER-6. Natural Environmental Research Program, Plymouth.

Clarke, T.A. - 1973. Some aspects of the ecology of lanternfishes (Myctophidae) in the Pacific Ocean near Hawaii. Fish. Bull., 71: $401-434$

Cowen, R.K. and L.R. Castro. - 1994. Relation of coral reef fish larval distributions to island scale circulation around Barbados, West Indies. Bull. Mar. Sci., 54: 228-244.

Cowen, R.K., C.B. Paris and A. Srinivasan. - 2006. Scaling of connectivity in marine populations. Science, $311: 522-527$.

Cushing, D.H. - 1990. Plankton production and year-class strength in fish populations: an update of the match/mismatch hypothesis. Adv. Mar. Biol., 26: 249-293.

De León, A.R. and J.G. Braun. - 1973. Annual cycle of primary production and its relation to nutrients in the Canary Islands waters. Bol. Inst. Esp. Oceanogr., 167: 1-24.

Franquet, F. and A. Brito. - 1995. Especies de interés pesquero de Canarias. Consejería de Pesca y Transportes del Gobierno de Canarias, Santa Cruz de Tenerife.

Goodyear, R.H., B.J. Zahuranec, W.L. Pugh and R.H. Gibbs - 1972. Ecology and vertical distribution of Mediterranean midwater fishes. Mediterranean Biological Studies. U.S. Navy Office, 91-229.

Hammer, W.M. and I.R. Hauri. - 1981. Effects of island mass: water flow and plankton pattern around a reef in the Great Barrie Reef lagoon, Australia. Limnol. Oceanogr., 26: 1084-1102.

Hernández-León, S. - 1988. Gradients of mesozooplankton biomass and ETS activity in the wind-shear area as evidence of an island mass effect in the Canary Island waters. J. Plankton Res., 10:
1141-1154.

Hernández-León, S. - 1998. Annual cycle of epiplanktonic copepods in Canary Island waters. Fish. Oceanogr., 7: 252-257.

Hernández-León, S. - 2008. Natural variability of fisheries and lunar illumination: a hypothesis. Fish Fish., 9: 1-17.

Hernández-León, S., C. Almeida, L. Yebra, J. Arístegui, M. L. Fernández de Puelles and J. García-Braun. - 2001. Zooplankton abundance in subtropical waters: Is there a lunar cycle? Sci. Mar., 65: 59-63.

Hernández-León, S., C. Almeida, P. Bécognée, L. Yebra and J. Arístegui. - 2004. Zooplankton biomass and indices of grazing and metabolism during a late winter bloom in subtropical waters. Mar. Biol., 145: 1191-1200.

Hernández-León, S., M. Gómez and J. Arístegui. - 2007. Mesozooplankton in the Canary Current System: The coastal-ocean transition zone. Progr. Oceanogr., 74: 397-421.

Hjort, J. - 1914. Fluctuations in the great fisheries of northern Europe viewed in the light of biological research. Rapp. P.-v. Reun. Cons. Int. Explor. Mer., 20: 1-228.

Houde, E.D. - 1987. Fish early life dynamics and recruitment variability. Am. Fish. Soc. Symp. Ser., 2: 17-29.

Houde, E.D. - 2008. Emerging from Hjort's shadow. J. Northwest Atl. Fish. Sci., 41: 53-70.

Isari, S., N. Fragopoulu and S. Somarakis. - 2008. Interranual variability in horizontal patterns of larval fish assemblages in the northeastern Aegean Sea (eastern Mediterranean) during early summer. Est. Coast Shelf. Sci., 79: 607-619.

Jones, G.P., M.J. Millcich, M.J. Emsile and C. Lunow. - 1999. Selfrecruitment in a coral fish population. Nature, 402: 802-804.

La Violette, P.E. - 1974. A satellite-aircraft thermal study of the upwelled waters off Spanish Sahara. J. Phys. Oceanogr., 4: 676-684.

Landeira, J.M., F. Lozano-Soldevilla, S. Hernández-León and E.D. Barton. - 2009. Horizontal distribution of invertebrate larvae around the oceanic island of Gran Canaria: the effect of mesoscale variability. Sci. Mar., 73(4): 761-771.

Leis, J.M. - 1982. Nearshore distributional gradients of larval fish (15 taxa) and planktonic crustaceans (6 taxa) in Hawaii. Mar. Biol., 72: 89-97.

Leis, J.M. - 1991. The pelagic phase of coral reef fishes: larval biology of coral reef fishes. In: P.F. Sale (ed.), The ecology of fishes on coral reefs, pp. 183-230. Academic Press, San Diego.

Leis, J.M. - 2007. Behaviour as input for modelling dispersal of fish larvae: behaviour, biogeography, hydrodynamics, ontogeny, physiology and phylogeny meet hydrography. Mar. Ecol. Progr. Ser., 347: 185-193.

Loeb, V.J. - 1980. Patterns of spatial and species abundance within the larval fish assemblage of the North Pacific Central Gyre during late summer. Mar. Biol., 60: 189-200.

Longhurst, A.R. and D. Pauly. - 1987. Ecology of tropical oceans. Academic Press, San Diego.

Lorenzo, J.M. and J.G. Pajuelo. - 1996. Growth and reproductive biology of chub mackerel Scomber japonicus off the Canary Islands. S. Afr. J. Mar. Sci., 17: 275-280.

Lovegrove, T. - 1966. The determination of the dry weight of plankton and the effect of various factors on the values obtained. In: H. Barnes (ed.), Some contemporary studies in Marine Sciences, pp. 429-467. George Allen and Unwin Ltd., London.

Lowe-McConnell, R.H. - 1987. Ecological studies in tropical fish communities. Cambridge University Press, Cambridge, UK.

Moyano, M., J. Rodríguez and S. Hernández-León. - 2009. Larval fish abundance and distribution during the late winter bloom in the Canary Island waters. Fish. Oceanogr., 18: 51-61.

Muhling, B.A., L.E. Beckley, J.A. Koslow and A.F. Pearce. - 2008. Larval fish assemblages and water mass structure off the oligotrophic south-western Australian coast. Fish. Oceanogr., 17: 16-31.

Olivar, M.P. and I. Palomera. - 1994. Ontogeny and distribution of Hygophum benoiti (Pisces, Myctophidae) of the North Western Mediterranean. J. Plankton Res., 16: 977-991.

Pacheco, M. and A. Hernández-Guerra. - 1999. Seasonal variability of recurrent phytoplankton pigment patterns in the Canary Islands area. Int. J. Remote Sens., 20: 1405-1418.

Paris, C.B. and R.K. Cowen. - 2004. Direct evidence of a biophysical retention mechanism for coral reef fish larvae. Limnol. Oceanogr. 49: 1964-1979.

Queró, J.C., J.C. Hureau, C. Karrer, A. Post and L. Saldanha. - 1990. 
Check List of the fishes of the Eastern Tropical Atlantic. JNICT, Lisbon; SEI, Paris; and UNESCO, Paris.

Rodríguez, J.M. - 2000. Fish larvae from the Canary region in autumn. Sci. Mar., 64: 79-85.

Rodríguez, J.M., S. Hernández-León and E.D. Barton. - 1999. Mesoscale distribution of fish larvae in relation to an upwelling filament off Northwest Africa. Deep Sea Res. I, 46: 1969-1984.

Rodríguez, J.M., J.G. Braun and A. García. - 2000. Spatial variability of the mesozooplankton biomass and ichthyoplankton in the Canary region, in autumn 1991. J. Plankton Res., 22: 1377-1391.

Rodríguez, J.M., E.D. Barton, L. Eve and S. Hernández-León. - 2001. Mesozooplankton and ichthyoplankton distribution around Gran Canaria, an oceanic island in the NE Atlantic. Deep Sea Res. I, 48: 2161-2183.

Sabatés, A. - 1990. Distribution pattern of larval fish populations in the northwestern Mediterranean. Mar. Ecol. Prog. Ser., 59: 75-82.

Sabatés, A., P. Martín, J. Lloret and V. Raya. - 2006. Sea warming and fish distribution: the case of the small pelagic fish, Sardinella aurita, in the western Mediterraean. Glob. Change Biol.,
12: 2209-2219.

Sangrá, P., M. Auladell, A. Marrero-Díaz, J.L. Pelegrí, E. FraileNuez, A. Rodríguez-Santana, J.M. Martín, E. Mason and A. Hernández-Guerra. - 2007. On the nature of oceanic eddies shed by the Island of Gran Canaria. Deep Sea Res. I, 54: 687-709.

Smith, K.A. and I.M. Suthers. - 1999. Displacement of diverse ichthyoplankton assemblages by a coastal upwelling event on the Sydney shelf. Mar. Ecol. Progr. Ser., 176: 49-62.

Somarakis, S., P. Drakopoulos and V. Filippou. - 2002. Distribution and abundance of larval fish in the northern Aegean Sea eastern Mediterranean in relation to early summer oceanographic conditions. J. Plankton Res., 24: 339-357.

Swearer, S.E., J.E. Casselle, D.W. Lea and R.R. Warner. - 1999. Larval retention and recruitment in an island population of a coral-reef fish. Nature, 402: 799-802.

Yentsch, C.S. and D.W. Menzel. - 1963. A method for the determination of phytoplankton chlorophyll and phaeophytin fluorescence. Deep Sea Res. I, 10: 221-231.

Received November 15, 2008. Accepted June 25, 2009.

Published online October 5, 2009. 